Алгебра и анализ

Том 22 (2010), № 3
St. Petersburg Math. J.

Vol. 22 (2011), No. 3, Pages 393-410

S 1061-0022(2011)01148-3

Article electronically published on March 17, 2011

\title{
NON-HERMITIAN SPIN CHAINS WITH INHOMOGENEOUS COUPLING
}

\author{
A. G. BYTSKO
}

To Ludwig Dmitrievich Faddeev on his 75 th birthday

\begin{abstract}
An open $U_{q}\left(s l_{2}\right)$-invariant spin chain of spin $S$ and length $N$ with inhomogeneous coupling is investigated as an example of a non-Hermitian (quasi-Hermitian) model. For several particular cases of such a chain, the ranges of the deformation parameter $\gamma$ are determined for which the spectrum of the model is real. For a certain range of $\gamma$, a universal metric operator is constructed, and thus, the quasi-Hermitian nature of the model is established. This universal metric operator is nondynamical, its structure is determined only by the symmetry of the model. The results apply, in particular, to all known homogeneous $U_{q}\left(s l_{2}\right)$-invariant integrable spin chains with nearest-neighbor interaction. In addition, the most general form of a metric operator for a quasi-Hermitian operator in finite-dimensional spaces is discussed.
\end{abstract}

\section{INTRODUCTION}

A bounded linear operator $\mathrm{H}$ in a complex Hilbert space $\mathfrak{H}$ equipped with the inner product $\langle x, y\rangle$ is said to be symmetrizable if there exists a Hermitian operator $\eta$ such that $\eta \neq 0$ and

$$
\eta \mathrm{H}=\mathrm{H}^{*} \eta .
$$

Symmetrizable operators have been studied in the mathematical literature since long ago; see [Za, Re, He, Di, S1, S2]. Following Dieudonné Di], we shall say that a symmetrizable operator $\mathrm{H}$ is quasi-Hermitian if the symmetrizing operator $\eta$ is positive definite.

If $\eta$ is invertible, then a quasi-Hermitian operator $\mathrm{H}$ is similar to a Hermitian one, so that it has a real spectrum (the spectrum of $\mathrm{H}$ may not be purely real if $\eta$ is positive definite but not invertible; see [Di, S2]). This makes it possible to interpret [SGH] an irreducible set of quasi-Hermitian operators as quantum mechanical observables, provided that they share a common symmetrizing operator $\eta$. In this context $\eta$ is called a metric operator, because the observables become Hermitian operators with respect to the modified inner product $\langle x, y\rangle_{\eta} \equiv\langle x, \eta y\rangle$. Interesting motivating examples of non-Hermitian operators with real spectrum are the Hamiltonian of the lattice Reggeon field theory [CS, the Hamiltonian of the Ising quantum spin chain in an imaginary magnetic field Ge, the Hamiltonians of affine Toda field theories with an imaginary coupling constant $[\mathrm{Ho}$, and the Schrödinger operator with an imaginary cubic potential [BZ]. The last example was generalized BB2 to a large class of symmetrizable Hamiltonians possessing the PT (parity and time-reversal) symmetry and having, in accordance with Wigner's theory Wi] of antiunitary operators, (partially) real spectra. Since then, a great deal of research

2010 Mathematics Subject Classification. Primary 81T10.

Key words and phrases. Quasi-Hermitian Hamiltonians, quantum algebras, spin chains. 
in the physical literature has been devoted to symmetrizable and, in particular, quasiHermitian Hamiltonians, which led to the construction of numerous interesting examples and the (re)discovery of many mathematical aspects; see [Be, M2] for reviews.

The Hamiltonian $\mathrm{H}$ of a physical model is often given by the sum or, more generally, a linear combination of local Hamiltonians $\mathrm{H}_{n}, n=1, \ldots, N$, with real coefficients (coupling constants)

$$
\mathrm{H}=\sum_{n=1}^{N} a_{n} \mathrm{H}_{n}, \quad a_{n} \in \mathbb{R} .
$$

Here we face an immediate difficulty absent in the theory of Hermitian operators: no general criterion is known that would judge whether $\mathrm{H}$ is a quasi-Hermitian operator, given that all $\mathrm{H}_{n}$ are quasi-Hermitian operators (it is not assumed that they share a common symmetrizing operator). This problem arises naturally for Hamiltonians of various spin chains where the interaction between adjacent sites is described by quasi-Hermitian operators. For instance, the reality of spectra and the existence of metric operators for such compound chains were investigated for the Ising chain in an imaginary magnetic field $\mathrm{Ge}, \mathrm{CF}$, for the Jordanian twist of the Heisenberg chain [KS], and for the homogeneous XXZ model of spin $\frac{1}{2}[\mathrm{KW}]$. In the present paper we address the quasi-Hermiticity problem for an open spin chain of spin $S$ with nearest-neighbor Hamiltonians $\mathrm{H}_{n}$ that have the most general form respecting the $U_{q}\left(s l_{2}\right)$-symmetry.

The paper is organized as follows. In Subsection 1.1, we provide the necessary facts about quasi-Hermitian operators, and in Subsection 1.2, we discuss the most general form of a metric operator. In Subsection 2.1, we recall the basic notions related to the quantum algebra $U_{q}\left(s l_{2}\right)$, discuss the phenomenon of being non-Hermitian for the tensor product of its representations in the case of $q=e^{i \gamma}, \gamma \in \mathbb{R}$, and introduce an open $U_{q}\left(s l_{2}\right)$-invariant spin chain of length $N$ with inhomogeneous coupling. In Subsections 2.2 and 2.3, we investigate the reality of spectra of particular cases of such a chain for $N=3,4,5$ by considering the minimal polynomials of the corresponding Hamiltonians. Extrapolating our results, we formulate two conjectures on the range of $\gamma$ in which the spectrum is real. In Subsection 2.4, we construct a multiparametric family of universal (i.e., independent of coupling constants) symmetrizing operators for the most general $U_{q}\left(s l_{2}\right)$-invariant open spin chain with a nearest-neighbor interaction. The construction exploits solely the quantum algebraic symmetry of the model and is formulated in terms of related algebraic objects such as the $R$-matrix and the comultiplication. For a one-parameter subfamily of symmetrizing operators, we determine the range of $\gamma$ in which it contains positive definite operators, and thus, the Hamiltonian of the model is quasi-Hermitian. In the Conclusion, we summarize and briefly discuss our results. The Appendix contains proofs of some statements given in the main text and some technical details on $R$-matrices and projections to irreducible subspaces in tensor products.

\section{§1. Quasi-Hermitian operators and metric operators}

1.1. Preliminaries. Consider the eigenvalue problem for a quasi-Hermitian operator $\mathrm{H}$,

$$
\mathrm{H} \omega_{j}=\lambda_{j} \omega_{j}, \quad\left\langle\omega_{j}, \omega_{j}\right\rangle=1 .
$$

Let $\left\{\omega_{j}\right\}$ be the set of normalized eigenvectors of $\mathrm{H}$, and $\operatorname{Spec}(\mathrm{H}) \equiv\left\{\lambda_{j}\right\}$ the set of the corresponding eigenvalues. Here and below we shall restrict our consideration to the case of a finite-dimensional Hilbert space, $d \equiv \operatorname{dim} \mathfrak{H}<\infty$. In this case, the metric operator $\eta$ is invertible and the quasi-Hermitian operator $\mathrm{H}$ is similar to the Hermitian operator

$\eta^{\frac{1}{2}} \mathrm{H} \eta^{-\frac{1}{2}}$, whence it is immediate that $\operatorname{Spec}(\mathrm{H}) \subset \mathbb{R}$, and the set $\left\{\omega_{j}\right\}$ is a complete set of vectors in $\mathfrak{H}$. 
Remark 1. The converse is also true; see [S1, Theorem 3.3]: if a linear operator $\mathrm{H}$ in a finite-dimensional complex Hilbert space $\mathfrak{H}$ has a real spectrum and the set $\left\{\omega_{j}\right\}$ of its eigenvectors is complete, then $\mathrm{H}$ is quasi-Hermitian. A metric operator for a given $\mathrm{H}$ can be constructed as follows (see, e.g., [M1]): take an arbitrary orthonormal basis $\left\{e_{j}\right\}$ in $\mathfrak{H}$ and define a linear operator $\Omega$ such that $\Omega \omega_{j}=e_{j}$. Then $\Omega$ is invertible and $\mathrm{H}_{0}=\Omega \mathrm{H} \Omega^{-1}$ is Hermitian. It follows that $\eta_{0}=\Omega^{*} \Omega$ is a metric operator for $\mathrm{H}$. Note that, actually, $\eta_{0}$ does not depend on the choice of the basis $\left\{e_{j}\right\}$.

Remark 2. In the physical literature on PT-symmetric models [BBJ, $\mathrm{Be}, \mathrm{M} 2, \mathrm{AF}$, the pseudo-Hermitian operators are also considered, i.e., the symmetrizable operators for which $\eta$ is invertible but not positive definite. The pseudo-Hermitian nature of $\mathrm{H}$ implies only that if $\lambda \in \operatorname{Spec}(\mathrm{H})$, then $\bar{\lambda} \in \operatorname{Spec}(\mathrm{H})$, as, e.g., the case were $\mathrm{H}=\left(\begin{array}{cc}i & 0 \\ 0 & -i\end{array}\right), \eta=\left(\begin{array}{ll}0 & 1 \\ 1 & 0\end{array}\right)$. Furthermore, the set of the eigenvectors of a pseudo-Hermitian operator is not necessarily a complete set of vectors in $\mathfrak{H}$, as another simple example demonstrates: $\mathbf{H}=\left(\begin{array}{ll}1 & 1 \\ 0 & 1\end{array}\right)$, $\eta=\left(\begin{array}{ll}0 & 1 \\ 1 & 0\end{array}\right)$.

The eigenvectors $\left\{\omega_{j}\right\}$ of a quasi-Hermitian operator $\mathrm{H}$ provide a nonorthogonal basis in $\mathfrak{H}$. Consider the corresponding Gram matrix $G$ with the entries $G_{k n}=\left\langle\omega_{k}, \omega_{n}\right\rangle$. The matrix $G$ is invertible, Hermitian (with respect to the conjugate transpose operation), and positive definite. The set of vectors $\left\{\widetilde{\omega}_{j}\right\}$, where $\widetilde{\omega}_{j}=\sum_{n=1}^{d}\left(G^{-1}\right)_{n j} \omega_{n}$, provides another nonorthogonal basis in $\mathfrak{H}$. Its Gram matrix is $G^{-1}$. The bases $\left\{\omega_{j}\right\}$ and $\left\{\widetilde{\omega}_{j}\right\}$ form a biorthogonal system:

$$
\left\langle\omega_{k}, \omega_{j}\right\rangle=\delta_{k j}, \quad\left\langle\omega_{k}, \widetilde{\omega}_{j}\right\rangle=\delta_{k j}, \quad\left\langle\widetilde{\omega}_{k}, \widetilde{\omega}_{j}\right\rangle=\left(G^{-1}\right)_{k j} .
$$

Remark 3. Note that, in general, the $\widetilde{\omega}_{j}$ are not normalized. Indeed, the positive definiteness of $G^{-1}$ implies only that $\left(G^{-1}\right)_{j j}>0$ for all $j$.

Any vector $x \in \mathfrak{H}$ gives rise to a linear functional $x^{\dagger}: \mathfrak{H} \mapsto \mathbb{C}$ such that $x^{\dagger}(y)=\langle x, y\rangle$. Since $\left\{\omega_{j}\right\}$ and $\left\{\widetilde{\omega}_{j}\right\}$ are bases in $\mathfrak{H}$, any linear operator $A$ acting in $\mathfrak{H}$ can be written in the form

$$
\mathrm{A}=\sum_{k, n=1}^{d} O(\mathrm{~A})_{k n} \omega_{k} \omega_{n}^{\dagger}=\sum_{k, n=1}^{d} \widetilde{O}(\mathrm{~A})_{k n} \widetilde{\omega}_{k} \widetilde{\omega}_{n}^{\dagger}
$$

where $O(\mathrm{~A})$ and $\widetilde{O}(\mathrm{~A})$ are complex matrices (we call them the symbols of $\mathrm{A})$. It is useful to observe that $O\left(\mathrm{~A}^{*}\right)=(O(\mathrm{~A}))^{*}, \widetilde{O}\left(\mathrm{~A}^{*}\right)=(\widetilde{O}(\mathrm{~A}))^{*}$, and

$$
\begin{aligned}
O(\mathrm{AB}) & =O(\mathrm{~A}) G O(\mathrm{~B}), & & \widetilde{O}(\mathrm{~A} \mathrm{~B})=\widetilde{O}(\mathrm{~A}) G^{-1} \widetilde{O}(\mathrm{~B}), \\
\widetilde{O}(\mathrm{~A}) & =G O(\mathrm{~A}) G, & & \widetilde{O}(\mathrm{~A}) O\left(\mathrm{~A}^{-1}\right)=E,
\end{aligned}
$$

where $E$ is the identity matrix, and the last relation makes sense if $A$ is invertible.

Let $\mathrm{P}_{j}$ and $\widetilde{\mathrm{P}}_{j}$ denote projectors in $\mathfrak{H}$ to $\omega_{j}$ and $\widetilde{\omega}_{j}$, respectively, i.e., $\mathrm{P}_{j} \omega_{k}=\delta_{j k} \omega_{j}$ and $\widetilde{\mathbf{P}}_{j} \widetilde{\omega}_{k}=\delta_{j k} \widetilde{\omega}_{j}$. Relations (4) imply that these projectors are given by

$$
\mathrm{P}_{j}=\omega_{j} \widetilde{\omega}_{j}^{\dagger}=\sum_{n=1}^{d}\left(G^{-1}\right)_{j n} \omega_{j} \omega_{n}^{\dagger}=\sum_{n=1}^{d} G_{n j} \widetilde{\omega}_{n} \widetilde{\omega}_{j}^{\dagger}, \quad \widetilde{\mathrm{P}}_{j}=\mathrm{P}_{j}^{*}=\widetilde{\omega}_{j} \omega_{j}^{\dagger} .
$$

The resolutions of the unity, $\sum_{j=1}^{d} \mathrm{P}_{j}=1=\sum_{j=1}^{d} \mathrm{P}_{j}^{*}$, are due to the completeness of the sets $\left\{\omega_{j}\right\}$ and $\left\{\widetilde{\omega}_{j}\right\}$.

1.2. General form of the metric operator. Consider a quasi-Hermitian operator $\mathbf{H}$ that has $d^{\prime} \leq d$ distinct eigenvalues $\left\{\lambda_{j}\right\}$ with multiplicities $\mu_{j} \geq 1$, so that we have $\sum_{j=1}^{d^{\prime}} \mu_{j}=d$. The eigenvectors corresponding to a given eigenvalue $\lambda_{j}$ span the subspace 
$\mathfrak{H}_{j} \subset \mathfrak{H}$. Let $\left\{\omega_{j, k}\right\}, k=1, \ldots, \mu_{j}$, be a basis of $\mathfrak{H}_{j}$ (not unique if $\mu_{j}>1$ ), and let $\mathrm{P}_{j, k}$ denote the projector onto $\omega_{j, k}$.

Proposition 1. a) For a quasi-Hermitian operator $\mathrm{H}$ that has the spectrum $\left\{\lambda_{j}\right\}$ with multiplicities $\mu_{j}$, fix some basis $\left\{\omega_{j, k}\right\}$ in each subspace $\mathfrak{H}_{j}$. Then, for this $\mathrm{H}$, the most general form of a metric operator and its inverse is as follows:

$$
\eta=\sum_{j=1}^{d^{\prime}} \sum_{k, n=1}^{\mu_{j}}\left(\Phi_{j}\right)_{k n} \widetilde{\omega}_{j, k} \widetilde{\omega}_{j, n}^{\dagger}, \quad \eta^{-1}=\sum_{j=1}^{d^{\prime}} \sum_{k, n=1}^{\mu_{j}}\left(\Phi_{j}^{-1}\right)_{k n} \omega_{j, k} \omega_{j, n}^{\dagger},
$$

where the $\Phi_{j}$ are arbitrary Hermitian positive definite matrices of size $\mu_{j} \times \mu_{j}$.

b) For a quasi-Hermitian operator $\mathrm{H}$ that has the spectrum $\left\{\lambda_{j}\right\}$ with multiplicities $\mu_{j}$, take some metric operator $\eta$. Then there exists a choice of bases $\left\{\omega_{j, k}\right\}$ of subspaces $\mathfrak{H}_{j}$ such that the given operator $\eta$ and its inverse have the form

$$
\eta=\sum_{j=1}^{d^{\prime}} \sum_{k=1}^{\mu_{j}} \Phi_{j, k} \mathrm{P}_{j, k}^{*} \mathrm{P}_{j, k}, \quad \eta^{-1}=\sum_{j=1}^{d^{\prime}} \sum_{k=1}^{\mu_{j}} \widetilde{\Phi}_{j, k} \mathrm{P}_{j, k} \mathrm{P}_{j, k}^{*},
$$

where the $\Phi_{j, k}$ are arbitrary positive numbers and $\widetilde{\Phi}_{j, k}=\left(\left(G^{-1}\right)_{\{j, k\},\{j, k\}} \Phi_{j, k}\right)^{-1}$.

Remark 4. It is natural to regard metric operators differing only by a positive constant scalar factor as equivalent. Thus, formulas (10) describe $(d-1)$-parameter families of operators. If the spectrum of a quasi-Hermitian operator $\mathrm{H}$ is simple, then these formulas give the most general form of the corresponding metric operator and its inverse.

Remark 5. As noted in the preceding remark, parts a) and b) of Proposition 1 are merely different forms of one and the same statement if the spectrum of $\mathrm{H}$ is simple. The difference arises if the spectrum of $\mathrm{H}$ is degenerate. Indeed, although any given metric operator can be brought to the form (10) that involves only projectors to the eigenvectors of $\mathrm{H}$, this requires a change of the basis in the Hilbert space after we have chosen the metric operator. But if we work with a fixed basis, then the most general form of a metric operator (9) cannot in general be rewritten only in terms of projectors to the eigenvectors of $\mathrm{H}$ if it has a degenerate spectrum. This is so because $\mathrm{P}_{j, k}^{*} \mathrm{P}_{j, n}=G_{\{j, k\},\{j, n\}} \widetilde{\omega}_{j, k} \widetilde{\omega}_{j, n}^{\dagger}$, and the corresponding entry of the Gram matrix can be zero. (In fact, it is always zero if we choose an orthonormal basis in the subspace $\mathfrak{H}_{j}$.)

Remark 6. If all $\Phi_{j}$ are identity matrices, then (9) yields the operator $\eta_{0}$ considered in Remark 1. Indeed, it is easily seen that $\Omega^{-1}=\sum_{j=1}^{d} \omega_{j} e_{j}^{\dagger}$, whence $\eta_{0}^{-1}=\Omega^{-1}\left(\Omega^{*}\right)^{-1}=$ $\sum_{j=1}^{d} \omega_{j} \omega_{j}^{\dagger}$.

Remark 7. If $\mathrm{H}$ has a simple spectrum, we can use equations (53) to rewrite formulas (10) in a form that does not involve eigenvectors explicitly:

$$
\begin{aligned}
\eta & =\sum_{j=1}^{d} \Theta_{j}\left(\prod_{n \neq j}^{d}\left(\mathbf{H}^{*}-\lambda_{n} 1\right)\right)\left(\prod_{m \neq j}^{d}\left(\mathbf{H}-\lambda_{m} 1\right)\right), \\
\eta^{-1} & =\sum_{j=1}^{d} \widetilde{\Theta}_{j}\left(\prod_{m \neq j}^{d}\left(\mathbf{H}-\lambda_{m} 1\right)\right)\left(\prod_{n \neq j}^{d}\left(\mathbf{H}^{*}-\lambda_{n} 1\right)\right),
\end{aligned}
$$

where the $\Theta_{j}$ are arbitrary positive numbers, and $\widetilde{\Theta}_{j}=\left(\left(G^{-1}\right)_{j j} \Theta_{j}\right)^{-1}$.

As an example, consider the following operator acting in $\mathbb{C}^{2}$ (it is related to the Hamiltonian (93) in $\mathrm{Be}$ by a change of variables that ensures the reality of the spectrum):

$$
\mathbf{H}=\left(\begin{array}{cc}
e^{i \theta} \sinh z & \sin \theta \cosh z \\
\sin \theta \cosh z & e^{-i \theta} \sinh z
\end{array}\right)=(\sinh z) e^{i \theta \sigma_{3}}+(\sin \theta \cosh z) \sigma_{\mathbf{1}}, \quad \theta, z \in \mathbb{R}
$$


Here and below we use the standard notation for the Pauli matrices: $\sigma_{1}=\left(\begin{array}{ll}0 & 1 \\ 1 & 0\end{array}\right), \sigma_{2}=$ $\left(\begin{array}{cc}0 & -i \\ i & 0\end{array}\right), \sigma_{3}=\left(\begin{array}{cc}1 & 0 \\ 0 & -1\end{array}\right)$. The operator (13) is not Hermitian, but it has real eigenvalues $\lambda_{ \pm}=\cos \theta \sinh z \pm \sin \theta$. Observe that its spectral resolution can be written in the form

$$
\mathrm{H}=\lambda_{+} \mathrm{P}_{+}+\lambda_{-} \mathrm{P}_{-}, \quad \mathrm{P}_{ \pm}=e^{-\frac{z}{2} \sigma_{2}} \frac{\left(1 \pm \sigma_{1}\right)}{2} e^{\frac{z}{2} \sigma_{2}},
$$

which makes it obvious that $\mathrm{H}=\Omega^{-1} \mathrm{H}_{0} \Omega$, where $\Omega=e^{\frac{z}{2} \sigma_{2}}$ and $\mathrm{H}_{0}$ is Hermitian. Consequently, by Remark 11. we have $\eta_{0}=\Omega^{*} \Omega=e^{z \sigma_{2}}$, whereas (10) yields a oneparameter family of metric operators. Namely, taking $\Phi_{ \pm}=e^{ \pm \varphi} / \cosh z$, where $\varphi \in \mathbb{R}$, we obtain

$$
\eta_{\varphi}=e^{\frac{z}{2} \sigma_{2}} e^{\varphi \sigma_{1}} e^{\frac{z}{2} \sigma_{2}}
$$

In this form, the positive definiteness of $\eta_{\varphi}$ is self-evident, and we recover $\eta_{0}$ for $\varphi=0$.

\section{$\S 2$. Spin Chains With InHOMOGENEOUS COUPLing}

2.1. Spin chains with $U_{q}\left(s l_{2}\right)$-symmetry. We shall consider one-dimensional lattice models (open chains with free boundary conditions) that have $U_{q}\left(s l_{2}\right)$-symmetry. Recall that the algebra $U_{q}\left(s l_{2}\right)$ has the following defining relations:

$$
[E, F]=\frac{K^{2}-K^{-2}}{q-q^{-1}}, \quad K E=q E K, \quad K F=q^{-1} F K .
$$

A comultiplication consistent with these relations can be chosen as follows:

$$
\Delta(E)=E \otimes K^{-1}+K \otimes E, \quad \Delta(F)=F \otimes K^{-1}+K \otimes F, \quad \Delta(K)=K \otimes K .
$$

Let $S$ be a positive integer or semi-integer, and let $q=e^{i \gamma}$, where $\gamma \in \mathbb{R}$ and $2 S|\gamma|<\pi$. Let $V^{S} \simeq \mathbb{C}^{2 S+1}$ be an irreducible highest weight $U_{q}\left(s l_{2}\right)$-module, and $\left\{\omega_{k}\right\}_{k=-S}^{S}$ its canonical orthonormal basis in which $K$ is diagonalized. We consider the standard representation $\pi_{S}$ of $U_{q}\left(s l_{2}\right)$ on $V^{S}$ :

$$
\begin{aligned}
& \pi_{S}(E) \omega_{k}=\sqrt{[S-k][S+k+1]} \omega_{k+1}, \quad \pi_{S}(K) \omega_{k}=q^{k} \omega_{k}, \\
& \pi_{S}(F) \omega_{k}=\sqrt{[S+k][S-k+1]} \omega_{k-1},
\end{aligned}
$$

where $[t] \equiv \frac{\sin \gamma t}{\sin \gamma}$. In particular,

$$
\pi_{\frac{1}{2}}(E)=\sigma^{+} \equiv \frac{1}{2}\left(\sigma_{1}+i \sigma_{2}\right), \quad \pi_{\frac{1}{2}}(F)=\sigma^{-} \equiv \frac{1}{2}\left(\sigma_{1}-i \sigma_{2}\right), \quad \pi_{\frac{1}{2}}(K)=e^{i \frac{\gamma}{2} \sigma_{3}} .
$$

For $2 S|\gamma|<\pi$, the nonzero matrix entries of $\pi_{S}(E)$ and $\pi_{S}(F)$ are positive, and these matrices are conjugate transposes of each other. Therefore, formulas (18) can be regarded as a representation of the algebra $U_{q}\left(s l_{2}\right)$ with the anti-involution

$$
E^{*}=F, \quad F^{*}=E, \quad K^{*}=K^{-1} .
$$

However, the algebra $U_{q}\left(s l_{2}\right)$ with such an anti-involution is not a Hopf $*$-algebra, i.e., $(\Delta(X))^{*} \neq \Delta\left(X^{*}\right)$ in general. Instead we have $(\Delta(X))^{*}=\mathbb{P} \Delta\left(X^{*}\right) \mathbb{P}$, where $\mathbb{P}$ is the operator of permutation of the tensor factors in $U_{q}\left(s l_{2}\right)^{\otimes 2}$. This is the origin of the non-Hermitian nature of models to be considered below.

The comultiplication (17) determines the decomposition $V^{S} \otimes V^{S}=\bigoplus_{s=0}^{2 S} V^{s}$, where each $V^{s}$ is an irreducible $U_{q}\left(s l_{2}\right)$-submodule. The inner product on $V^{S}$ gives rise to an inner product on $V^{S} \otimes V^{S}:\left\langle\omega_{k} \otimes \omega_{m}, \omega_{k^{\prime}} \otimes \omega_{m^{\prime}}\right\rangle=\delta_{k k^{\prime}} \delta_{m m^{\prime}}$. A basis for $V^{S} \otimes V^{S}$ can be taken to be $\left\{\omega_{s, k}\right\}$, where $s=0, \ldots, 2 S$, and, for a given $s$, the vectors $\omega_{s, k}$, $k=-S, \ldots, S$ comprise the canonical basis of $V^{s}$. 
An important difference between the cases where $q \in \mathbb{R}$ and $|q|=1$ is that in the latter case vectors from different submodules can be nonorthogonal. For instance, the basis for $V^{\frac{1}{2}} \simeq \mathbb{C}^{2}$ is $\omega_{\frac{1}{2}}=\left(\begin{array}{l}1 \\ 0\end{array}\right), \omega_{-\frac{1}{2}}=\left(\begin{array}{l}0 \\ 1\end{array}\right)$, and the basis for $V^{\frac{1}{2}} \otimes V^{\frac{1}{2}}=V^{0} \oplus V^{1}$ is

$$
\omega_{0,0}=\frac{1}{\sqrt{\varkappa}}\left(\begin{array}{c}
0 \\
q^{-\frac{1}{2}} \\
-q^{\frac{1}{2}} \\
0
\end{array}\right), \quad \omega_{1,1}=\left(\begin{array}{l}
1 \\
0 \\
0 \\
0
\end{array}\right), \quad \omega_{1,0}=\frac{1}{\sqrt{\varkappa}}\left(\begin{array}{c}
0 \\
q^{\frac{1}{2}} \\
q^{-\frac{1}{2}} \\
0
\end{array}\right), \quad \omega_{1,-1}=\left(\begin{array}{l}
0 \\
0 \\
0 \\
1
\end{array}\right) .
$$

For $q \in \mathbb{R}$, these vectors are orthogonal, and normalization requires setting $\varkappa=[2]$. For $|q|=1$, the vectors are normalized if $\varkappa=2$, and we have $\left\langle\omega_{0,0}, \omega_{1,0}\right\rangle=i \sin \gamma$.

Remark 8. Basis vectors from different submodules can be nonorthogonal only if they have equal eigenvalues under the action of $\mathrm{K}_{12}=\left(\pi_{S} \otimes \pi_{S}\right) \Delta(K)$. Indeed, relations (17) and (19) show that $\mathrm{K}_{12}$ is unitary, $\mathrm{K}_{12}^{*}=\mathrm{K}_{12}^{-1}$. Therefore, if $\mathrm{K}_{12} \omega=q^{k} \omega$ and $\mathrm{K}_{12} \omega^{\prime}=$ $q^{k^{\prime}} \omega^{\prime}$, then $\left\langle\omega^{\prime}, \mathrm{K}_{12} \omega\right\rangle=q^{k}\left\langle\omega^{\prime}, \omega\right\rangle$, whence $q^{-k}\left\langle\omega, \omega^{\prime}\right\rangle=\left\langle\omega, \mathrm{K}_{12}^{*} \omega^{\prime}\right\rangle=\left\langle\omega, \mathrm{K}_{12}^{-1} \omega^{\prime}\right\rangle=$ $q^{-k^{\prime}}\left\langle\omega, \omega^{\prime}\right\rangle$, which implies that $q^{k}=q^{k^{\prime}}$ if $\left\langle\omega, \omega^{\prime}\right\rangle \neq 0$.

Let $\mathrm{P}^{S, s}$ denote the projector onto the irreducible submodule $V^{s}$ in $V^{S} \otimes V^{S}$. Some details on the structure of these projectors are given in Appendix 3.2. In particular, the projectors $\mathrm{P}^{S, s}$ are not Hermitian, but they are symmetrizable operators:

$$
\left(\mathrm{P}^{S, s}\right)^{*}=\left.\mathrm{P}^{S, s}\right|_{q \rightarrow \bar{q}}=\mathbb{P} \mathrm{P}^{S, s} \mathbb{P} .
$$

In fact, by Remark 1, it is obvious that these projectors are quasi-Hermitian operators.

Consider a one-dimensional lattice with $N$ nodes, each node carrying an irreducible module $V^{S}$ as a local Hilbert space. For an operator $\mathrm{A}$ in $V^{S}$ or in $\left(V^{S}\right)^{\otimes 2}$, we use the standard notation $\mathrm{A}_{n}$ and $\mathrm{A}_{n m}$ for its embedding in operators in $\mathfrak{H}=\left(V^{S}\right)^{\otimes N}$ that act nontrivially only in the $n$th or in the $n$th and $m$ th tensor components, respectively. The operator

$$
\mathrm{H}_{\left\{a_{1}, \ldots, a_{N-1}\right\}}^{S, s}=\sum_{n=1}^{N-1} a_{n} \mathrm{P}_{n, n+1}^{S, s}, \quad a_{n} \in \mathbb{R},
$$

can be regarded as the Hamiltonian of an open spin chain with inhomogeneous coupling. This Hamiltonian commutes with the global action of $U_{q}\left(s l_{2}\right)$ in $\mathfrak{H}$; i.e., we have (see Appendix 3.2)

$$
\left[\mathrm{H}_{\left\{a_{1}, \ldots, a_{N-1}\right\}}^{S, s}, \pi_{S}^{\otimes N}\left(\Delta^{(N-1)}(X)\right)\right]=0, \quad \text { for any } X \in U_{q}\left(s l_{2}\right) .
$$

Here and in what follows we use the abbreviation $\pi_{S}^{\otimes N} \equiv\left(\pi_{S} \otimes \cdots \otimes \pi_{S}\right)$.

Recall that the positive integral power of the comultiplication used in (23) is defined recursively: $\Delta^{(1)} \equiv \Delta$ and $\Delta^{(N)}=\Delta_{N, n} \circ \Delta^{(N-1)}$. We denote $\Delta_{N, n} \equiv \operatorname{id}_{n-1} \otimes \Delta \otimes \operatorname{id}_{N-n}$, where $n$ can be any number from 1 to $N$, by the coassociativity of $\Delta$, i.e., $\Delta_{2,1} \circ \Delta=$ $\Delta_{2,2} \circ \Delta$.

Remark 9. The Hamiltonian (22) is pseudo-Hermitian in the homogeneous case (where $\left(a_{1}=\cdots=a_{N-1}\right)$ for any $N$ and in the two-periodic case $\left(a_{2 n+1}=a_{1}, a_{2 n}=a_{2}\right)$ for even $N$. The symmetrizing operator for these cases is given by $\eta=\mathbb{P}_{1, N} \mathbb{P}_{2, N-1} \ldots$.

In general, a lattice model with the Hamiltonian (22) is not integrable. However, its homogeneous case is integrable for $s=0$. The corresponding R-matrix is constructed by a Baxterization of the Temperley-Lieb algebra (see, e.g., $\mathrm{Ku}$ ). In particular, for $S=\frac{1}{2}$ and $s=0$, setting $a_{1}=a_{2}=\cdots=-\cos \gamma$, we recover the Hamiltonian of the well-known XXZ model of spin $\frac{1}{2}$ (which is an integrable deformation of the Heisenberg chain),

$$
\mathrm{H}_{\{-\cos \gamma, \ldots\}}^{\frac{1}{2}, 0}=\sum_{n=1}^{N-1}\left(\frac{1}{2}\left(\sigma_{n}^{+} \sigma_{n+1}^{-}+\sigma_{n}^{-} \sigma_{n+1}^{+}\right)+\frac{\cos \gamma}{4}\left(\sigma_{n}^{3} \sigma_{n+1}^{3}-1\right)+\frac{i \sin \gamma}{4}\left(\sigma_{n}^{3}-\sigma_{n+1}^{3}\right)\right) .
$$


2.2. $N=2$ and $N=3$. We commence by studying spectra of short chains. Since $\mathfrak{H}$ is finite-dimensional, we have $\operatorname{Spec} \mathrm{H}=\left\{\lambda: \mathcal{P}_{\mathrm{H}}(\lambda)=0\right\}$, where $\mathcal{P}_{\mathrm{H}}(\lambda)$ is the minimal polynomial for $\mathrm{H}$, i.e., the least degree nonzero polynomial such that $\mathcal{P}_{\mathrm{H}}(\mathrm{H})=0$. In the simplest case, $N=2$, we have $\mathrm{H}_{\left\{a_{1}\right\}}^{S, s}=a_{1} \mathrm{P}_{12}^{S, s}$. The corresponding minimal polynomial is $\mathcal{P}_{a_{1}}^{S, s}(\lambda)=\lambda^{2}-a_{1} \lambda$, which shows that the spectrum consists of the points 0 and $a_{1}$, so that it is real.

For $N=3$, we have $\mathrm{H}_{\left\{a_{1}, a_{2}\right\}}^{S, s}=a_{1} \mathrm{P}_{12}^{S, s}+a_{2} \mathrm{P}_{23}^{S, s}$. First, we treat the case of $s=0$. In this case the projectors satisfy the relations of the Temperley-Lieb algebra BB1, B2]:

$$
\mathrm{P}_{n-1, n}^{S, 0} \mathrm{P}_{n, n+1}^{S, 0} \mathrm{P}_{n-1, n}^{S, 0}=\mu_{S} \mathrm{P}_{n-1, n}^{S, 0}, \quad \mu_{S}=\frac{1}{[2 S+1]^{2}} .
$$

Using these relations (see Appendix [3.3), we find the minimal polynomial for $\mathrm{H}_{\left\{a_{1}, a_{2}\right\}}^{S, 0}$ :

$$
\mathcal{P}_{a_{1}, a_{2}}^{S, 0}(\lambda)=\lambda\left(\lambda^{2}-\left(a_{1}+a_{2}\right) \lambda+a_{1} a_{2}\left(1-\mu_{S}\right)\right) .
$$

Hence, all eigenvalues of $\boldsymbol{H}_{\left\{a_{1}, a_{2}\right\}}^{S, 0}$ are real if and only if $\mathcal{D}^{S, 0} \equiv\left(a_{1}-a_{2}\right)^{2}+4 a_{1} a_{2} \mu_{S}$ is nonnegative, that is, if and only if

$$
\left(\frac{\sin (2 S+1) \gamma}{\sin \gamma}\right)^{2} \geq-\frac{4 a_{1} a_{2}}{\left(a_{1}-a_{2}\right)^{2}}
$$

Clearly, this condition is always fulfilled if $a_{1}$ and $a_{2}$ are both positive (or both negative). If $a_{1} a_{2}<0$, then the spectrum of $\mathrm{H}_{\left\{a_{1}, a_{2}\right\}}^{S, 0}$ is not real for the values of $\gamma$ where (27) fails. Note that the right-hand side of (27) attains the maximal value equal to 1 when $a_{2}=-a_{1}$. Hence, we infer that, even for $a_{1} a_{2}<0$, the spectrum of $\mathrm{H}_{\left\{a_{1}, a_{2}\right\}}^{S, 0}$ is guaranteed to be real for sufficiently small values of $\gamma$, namely, for $|\gamma|<\gamma_{S, 0}$, where

$$
\gamma_{S, 0}=\frac{\pi}{2(S+1)}
$$

is the minimal positive solution of the equation $\sin (2 S+1) \gamma=\sin \gamma$.

For $s \neq 0$, the projectors $\mathrm{P}^{S, s}$ do not satisfy relations of type (25). However, by evaluating (56) and (59) in the representation (18), one can find an explicit matrix form of these projectors and then search for the coefficients of the minimal polynomial for $\mathrm{H}_{\left\{a_{1}, a_{2}\right\}}^{S, s}$. The author performed these steps for $S=1, \frac{3}{2}$ and $s \leq 2 S$ using Mathematica $^{\mathrm{TM}}$. The polynomials obtained are:

$$
\mathcal{P}_{a_{1}, a_{2}}^{S, s}(\lambda)=\lambda^{\epsilon, s} \prod_{k}\left(\lambda^{2}-\left(a_{1}+a_{2}\right) \lambda+a_{1} a_{2}\left(1-d_{k}^{S, s}\right)\right),
$$

where the coefficients $d_{k}^{S, s}$ are listed in Appendix 3.4. In (29) we have $\epsilon_{S, s}=0$ if there is $d_{k}^{S, s}=1$ on the list for given $S$ and $s$ (which occurs for $s=2 S$ ), and $\epsilon_{S, s}=1$ otherwise.

From (29) we infer that all eigenvalues of $\mathrm{H}_{\left\{a_{1}, a_{2}\right\}}^{S, s}$ are real if and only if all $\mathcal{D}_{k}^{S, s} \equiv$ $\left(a_{1}-a_{2}\right)^{2}+4 a_{1} a_{2} d_{k}^{S, s}$ are nonnegative, that is, if and only if

$$
\left(d_{k}^{S, s}\right)^{-1} \geq-\frac{4 a_{1} a_{2}}{\left(a_{1}-a_{2}\right)^{2}} .
$$

Thus, we see that, for the values in question of $S$, the spectrum of $\mathrm{H}_{\left\{a_{1}, a_{2}\right\}}^{S, s}$ is real if $a_{1} a_{2}>0$ and is not real for some values of $\gamma$ if $a_{1} a_{2}<0$. In the latter case, the spectrum of $\mathbf{H}_{\left\{a_{1}, a_{2}\right\}}^{S, s}$ is guaranteed to be real for $|\gamma|<\gamma_{S, s}=\min _{k} \gamma_{S, s}^{\{k\}}$, where $\gamma_{S, s}^{\{k\}}$ is the minimal positive solution of the equation $d_{k}^{S, s}=1$. In Appendix 3.4 the coefficients $d_{k}^{S, s}$ are listed in such a way that $k=1$ corresponds to the minimal value among the $\gamma_{S, s}^{\{k\}}$. The list (64) of resulting values $\gamma_{S, s}$ together with formula (28) allows us to conjecture the following. 
Conjecture 1. For $a_{1} a_{2}<0$, the spectrum of $\mathrm{H}_{\left\{a_{1}, a_{2}\right\}}^{S, s}$ is real for $|\gamma|<\gamma_{S, s}$, where

$$
\gamma_{S, s}=\frac{\pi}{2\left(s+S+1-\delta_{s, 2 S}\right)} .
$$

Remark 10. Appearance of the correction for $s=2 S$ in (31) seems to be related to the fact that $\mathrm{P}^{S, 2 S}=1-\sum_{s \neq 2 S} \mathrm{P}^{S, s}$. In particular, (31) yields $\gamma_{\frac{1}{2}, 1}=\gamma_{\frac{1}{2}, 0}$, as should be anticipated, because $\mathrm{H}_{\left\{a_{1}, a_{2}\right\}}^{\frac{1}{2}, 1}$ and $\mathrm{H}_{\left\{a_{1}, a_{2}\right\}}^{\frac{1}{2}, 0}$ differ only by a sign and a shift by a real multiple of the identity operator.

2.3. $N=4$ and $N=5$ for $s=0$. For $N=4$ and $s=0$, a computation similar to that in Appendix 3.3 yields the following minimal polynomial:

$$
\begin{aligned}
& \mathcal{P}_{a_{1}, a_{2}, a_{3}}^{S, 0}(\lambda)=\lambda\left(\lambda^{2}-\left(a_{1}+a_{2}+a_{3}\right) \lambda+\left(a_{1}+a_{3}\right) a_{2}\left(1-\mu_{S}\right)\right) \\
& \quad \times\left(\lambda^{3}-\left(a_{1}+a_{2}+a_{3}\right) \lambda^{2}+\left(a_{1} a_{3}+a_{2}\left(a_{1}+a_{3}\right)\left(1-\mu_{S}\right)\right) \lambda-a_{1} a_{2} a_{3}\left(1-2 \mu_{S}\right)\right) .
\end{aligned}
$$

Analysis of the reality of the roots of the cubic factor is fairly complicated. Therefore, we restrict our consideration to the case where $a_{3}=a_{1}$ (which, in particular, includes the homogeneous case). In this case, (32) simplifies and acquires the following form:

$$
\begin{array}{r}
\mathcal{P}_{a_{1}, a_{2}, a_{1}}^{S, 0}(\lambda)=\lambda\left(\lambda-a_{1}\right)\left(\lambda^{2}-\left(a_{1}+a_{2}\right) \lambda+a_{1} a_{2}\left(1-2 \mu_{S}\right)\right) \\
\times\left(\lambda^{2}-\left(2 a_{1}+a_{2}\right) \lambda+2 a_{1} a_{2}\left(1-\mu_{S}\right)\right) .
\end{array}
$$

From (32) it follows that all eigenvalues of $\mathrm{H}_{\left\{a_{1}, a_{2}, a_{1}\right\}}^{S, 0}$ are real if and only if both $\widetilde{\mathcal{D}}_{1}^{S, 0} \equiv$ $\left(2 a_{1}-a_{2}\right)^{2}+8 a_{1} a_{2} \mu_{S}$ and $\widetilde{\mathcal{D}}_{2}^{S, 0} \equiv\left(a_{1}-a_{2}\right)^{2}+8 a_{1} a_{2} \mu_{S}$ are nonnegative. Thus, we conclude that the spectrum of $\mathrm{H}_{\left\{a_{1}, a_{2}, a_{1}\right\}}^{S, 0}$ is real if $a_{1} a_{2}>0$ and is not real for some values of $\gamma$ if $a_{1} a_{2}<0$. In the latter case, we note that $\widetilde{\mathcal{D}}_{1}^{S, 0}-\widetilde{\mathcal{D}}_{2}^{S, 0}=a_{1}\left(3 a_{1}-2 a_{2}\right)>0$. Therefore, for $a_{1} a_{2}<0$, the spectrum of $\mathbf{H}_{\left\{a_{1}, a_{2}, a_{1}\right\}}^{S, 0}$ is real if and only if $\widetilde{\mathcal{D}}_{2}^{S, 0}>0$, that is, if and only if

$$
\left(\frac{\sin (2 S+1) \gamma}{\sin \gamma}\right)^{2} \geq-\frac{8 a_{1} a_{2}}{\left(a_{1}-a_{2}\right)^{2}}
$$

The right-hand side of (33) attains its maximal value equal to 2 when $a_{2}=-a_{1}$. Thus, for $a_{1} a_{2}<0$, the spectrum of $\mathbf{H}_{\left\{a_{1}, a_{2}, a_{1}\right\}}^{S, 0}$ is guaranteed to be real for $|\gamma|<\widetilde{\gamma}_{S, 0}$, where $\tilde{\gamma}_{S, 0}$ is the minimal positive solution of the equation $\sin ^{2}(2 S+1) \gamma=2 \sin ^{2} \gamma$. Taking into account the fact that, for $S \geq \frac{1}{2}$, we have $\sin (2 S+1) \gamma / \sin \gamma>\sqrt{2}$ on some interval containing the point $\gamma=0$, the value $\widetilde{\gamma}_{S, 0}$ can be determined equivalently as the minimal positive solution of the equation

$$
U_{2 S}(\cos \gamma)=\sqrt{2},
$$

where $U_{n}(t)$ is the Chebyshev polynomial of the second kind $\left(U_{1}(t)=2 t, U_{2}(t)=4 t^{2}-1\right.$, etc.). In particular, we have

$$
\widetilde{\gamma}_{\frac{1}{2}, 0}=\frac{\pi}{4}, \quad \widetilde{\gamma}_{1,0}=\arccos \frac{\sqrt{1+\sqrt{2}}}{2} \approx 0.217 \pi .
$$

For $N=5$ and $s=0$, even in the reduced case where $a_{3}=a_{1}$ and $a_{4}=a_{2}$, the minimal polynomial $\mathcal{P}_{a_{1}, a_{2}, a_{1}, a_{2}}^{S, 0}(\lambda)$ contains factors that are polynomials in $\lambda$ of degree 4 or 5 . However, for $a_{1}=a_{3}=a, a_{2}=a_{4}=-a$, it simplifies and acquires the following form:

$$
\begin{aligned}
\mathcal{P}_{a,-a, a,-a}^{S, 0}(\lambda)= & \lambda\left(\lambda^{4}+a^{2}\left(3 \mu_{S}-2\right) \lambda^{2}+a^{4}\left(\mu_{S}^{2}-3 \mu_{S}+1\right)\right) \\
& \times\left(\lambda^{4}+a^{2}\left(6 \mu_{S}-5\right) \lambda^{2}+a^{4}\left(5 \mu_{S}^{2}-10 \mu_{S}+4\right)\right) .
\end{aligned}
$$


The first biquadratic factor here has only real roots if and only if $\mu_{S} \leq \frac{3-\sqrt{5}}{2}$. For this range of $\mu_{S}$, the second biquadratic factor also has only real roots. Thus, the spectrum of $\mathrm{H}_{\{a,-a, a,-a\}}^{S, 0}$ is guaranteed to be real for $|\gamma|<\widetilde{\gamma}_{S, 0}$, where $\widetilde{\gamma}_{S, 0}$ is the minimal positive solution of the equation $\sin (2 S+1) \gamma=\left(\frac{3+\sqrt{5}}{2}\right)^{1 / 2} \sin \gamma$, or equivalently, of the equation

$$
U_{2 S}(\cos \gamma)=\frac{1+\sqrt{5}}{2} \text {. }
$$

In particular, we have

$$
\widetilde{\gamma}_{\frac{1}{2}, 0}=\widetilde{\gamma}_{1,0}=\frac{\pi}{5}, \quad \widetilde{\gamma}_{\frac{3}{2}, 0} \approx 0.172 \pi
$$

Equations (28), (34), and (37) allow us to make the following conjecture about a chain with alternating coupling $\left(a_{1}=-a_{2}=a_{3}=-a_{4}=\cdots\right)$.

Conjecture 2. For an alternating chain with $N \geq 3$ nodes, the spectrum of $\mathrm{H}_{\{a,-a, a,-a, \ldots\}}^{S, 0}$ is real for $|\gamma|<\widetilde{\gamma}_{S, 0}$, where $\widetilde{\gamma}_{S, 0}$ is the minimal positive solution of the equation

$$
U_{2 S}(\cos \gamma)=2 \cos \frac{\pi}{N}
$$

Remark 11. For the alternating chain of spin $S=\frac{1}{2}$ and length $N$, equation (39) yields

$$
\widetilde{\gamma}_{\frac{1}{2}, 0}=\frac{\pi}{N}
$$

which is the most natural extrapolation of the values $\widetilde{\gamma}_{\frac{1}{2}, 0}$ given by (28), (35), and (38).

2.4. A universal metric operator. The most general form of a $\left.U_{q}\left(s l_{2}\right)\right)$-invariant open spin chain Hamiltonian with a nearest-neighbor interaction and an inhomogeneous coupling is the following:

$$
\mathrm{H}_{N}^{S}=\sum_{n=1}^{N-1} \sum_{s=0}^{2 S} b_{n, s} \mathrm{P}_{n, n+1}^{S, s}, \quad b_{n, s} \in \mathbb{R} .
$$

The Hamiltonian (22) considered previously is a particular case of (41) corresponding to the choice $b_{n, s^{\prime}}=a_{n} \delta_{s s^{\prime}}$. A particular homogeneous case of (41) corresponding to the choice $b_{n, s}=(\sin \gamma) \sum_{k=1}^{s} \cot (\gamma k)$ recovers the Hamiltonian of the integrable XXZ model of spin $S$ (see, e.g., B1]). For spin $S$ equal to 1, another integrable model recovered as a homogeneous case of (41) is the spin chain generated by the Izergin-Korepin R-matrix [K].

Now our aim is to construct a universal metric operator $\eta_{N}$ for the Hamiltonian (41), i.e., such that relation (11) holds true irrespective of the choice of the coupling coefficients $b_{n, s}$. As seen from equation (21), it suffices to find $\eta_{N}$ such that the relation

$$
\eta_{N} \mathrm{P}_{n, n+1}^{S, s}=\left(\mathrm{P}_{n, n+1}^{S, s}\right)^{*} \eta_{N}=\mathrm{P}_{n+1, n}^{S, s} \eta_{N}
$$

is valid for all $n=1, \ldots, N-1$.

Recall that the Hopf algebra $U_{q}\left(s l_{2}\right)$ is quasitriangular [D1]; i.e., it possesses a universal R-matrix that is an invertible element of (a completion of) $U_{q}\left(s l_{2}\right)^{\otimes 2}$ with the following properties:

$$
\begin{aligned}
R \Delta(X) & =\Delta^{\prime}(X) R \quad \text { for any } \quad X \in U_{q}\left(s l_{2}\right), \\
(\Delta \otimes \mathrm{id}) R & =R_{13} R_{23}, \quad(\mathrm{id} \otimes \Delta) R=R_{13} R_{12},
\end{aligned}
$$

where $\Delta^{\prime}(X) \equiv \mathbb{P} \Delta(X) \mathbb{P}$. In fact, there exist two universal R-matrices, because if $R^{+} \equiv$ $R$ satisfies (43)-(44), then so does $R^{-}=\mathbb{P}\left(R^{+}\right)^{-1} \mathbb{P}$. The explicit form of the universal R-matrices $R^{ \pm}$consistent with the comultiplication (17) is given in Appendix 3.5] 
We denote $\mathrm{R}^{ \pm} \equiv\left(\pi_{S} \otimes \pi_{S}\right) R^{ \pm}$. Equation (43) along with the fact that $\mathrm{P}^{S, s}$ is a function of $\left(\pi_{S} \otimes \pi_{S}\right) \Delta(C)$ (see (59)) implies that the projectors $\mathrm{P}^{S, s}$ are symmetrizable by $\mathrm{R}^{ \pm}$, i.e.,

$$
\mathrm{R}_{n, n+1}^{ \pm} \mathrm{P}_{n, n+1}^{S, s}=\mathrm{P}_{n+1, n}^{S, s} \mathrm{R}_{n, n+1}^{ \pm} .
$$

Equation (67) implies that $\eta_{2}^{S}(\alpha)=e^{i \alpha} \mathrm{R}^{+}+e^{-i \alpha} \mathrm{R}^{-}$is a Hermitian operator if $\alpha \in \mathbb{R}$. This, along with (45), means that $\eta_{2}^{S}(\alpha)$ is a one-parameter family of symmetrizing operators for a chain of length $N=2$. We shall extend this observation to a chain of arbitrary length as follows (a proof is given in Appendix 3.6).

Proposition 2. a) For a chain of length $N$, the following operators satisfy relations (42):

$$
\eta_{N}^{ \pm}=\overleftarrow{\mathrm{R}}_{N} \cdots \overleftarrow{\mathrm{R}}_{2}, \quad \text { where } \quad \overleftarrow{\mathrm{R}}_{n}=\mathrm{R}_{n-1, n} \cdots \mathrm{R}_{1, n}
$$

b) These operators can also be represented as follows:

$$
\eta_{N}^{ \pm}=\overrightarrow{\mathrm{R}}_{1} \cdots \overrightarrow{\mathrm{R}}_{N-1} \text {, where } \overrightarrow{\mathrm{R}}_{n}=\mathrm{R}_{n, n+1} \cdots \mathrm{R}_{n, N}
$$

c) These operators are conjugate to each other,

$$
\left(\eta_{N}^{+}\right)^{*}=\eta_{N}^{-} \text {. }
$$

Remark 12. The proof of Proposition2 is facilitated by the observation that the operation $\Delta^{ \pm} \equiv R^{ \pm} \Delta$ is coassociative (but note that it is not an algebra homomorphism) and that the operators (46) can be expressed in terms of its power: $\eta_{N}^{ \pm}=\pi_{S}^{\otimes_{N}}\left(\Delta_{ \pm}^{(N-1)}(1)\right)$; see Lemma 2.

As seen from (48), the symmetrizing operators $\eta_{N}^{ \pm}$are not Hermitian. However, we can utilize them to build a multi-parameter family of Hermitian symmetrizing operators as follows:

$$
\eta_{N}^{S}\left(\alpha_{1}, \ldots \mid \beta_{1}, \ldots\right)=\sum_{n \geq 1} \beta_{n}\left(e^{i \alpha_{n}} \eta_{N}^{+}\left(\left(\eta_{N}^{-}\right)^{-1} \eta_{N}^{+}\right)^{n-1}+e^{-i \alpha_{n}} \eta_{N}^{-}\left(\left(\eta_{N}^{+}\right)^{-1} \eta_{N}^{-}\right)^{n-1}\right),
$$

where all $\alpha_{n}$ and $\beta_{n}$ are real. Here we used a simple fact: if $\eta, \eta^{\prime}$, and $\eta^{\prime \prime}$ are symmetrizing operators for an operator $\mathrm{H}$, then so is $\eta\left(\eta^{\prime}\right)^{-1} \eta^{\prime \prime}$ if $\eta^{\prime}$ is invertible. In our case, $\eta_{N}^{ \pm}$are invertible because so are the universal R-matrices.

Note that, for $\gamma=0$, we have $\mathrm{R}^{ \pm}=1 \otimes 1$ and $\eta_{N}^{ \pm}=1_{N}$. Therefore, for sufficiently small values of $\gamma$ and appropriately chosen coefficients $\left\{\alpha_{n}\right\}$ and $\left\{\beta_{n}\right\}$, the operator (49) is positive definite, and thus, is a metric operator for the Hamiltonian (41).

For $\gamma \neq 0$, it is not straightforward to determine the values of $\left\{\alpha_{n}\right\}$ and $\left\{\beta_{n}\right\}$ for which (49) is positive definite. In the present article, we restrict our consideration to a one-parameter family,

$$
\eta_{N}^{S}(\alpha)=e^{i \alpha} \eta_{N}^{+}+e^{-i \alpha} \eta_{N}^{-}, \quad \alpha \in \mathbb{R} .
$$

Let $\gamma(\alpha)$ denote the maximal positive value of $\gamma$ for which (50) is positive definite for given $\alpha$, and let $\hat{\gamma}_{S} \equiv \sup _{\alpha} \gamma(\alpha)$. At least one of the eigenvalues of $\eta_{N}^{S}(\alpha)$ vanishes at $\gamma=\hat{\gamma}_{S}$. Therefore, $\hat{\gamma}_{S}$ can be determined from the condition $\operatorname{det}\left(\eta_{N}^{S}(\alpha)\right)=0$.

Lemma 1. We have

$$
\operatorname{det}\left(\eta_{N}^{S}(\alpha)\right)=\prod_{s=s_{\mathcal{o}}}^{S N}\left(e^{i \alpha} q^{s(s+1)-N S(S+1)}+e^{-i \alpha} q^{N S(S+1)-s(s+1)}\right)^{(2 s+1) \nu_{s}},
$$

where the $\nu_{s}$ are the multiplicities of the irreducible submodules in the decomposition $\left(V^{S}\right)^{\otimes N}=\bigoplus_{s=s_{\mathcal{o}}}^{N S} \nu_{s} V^{s}$. Here $s_{\mathcal{O}}=0$ if $N S$ is an integer and $s_{\mathcal{O}}=\frac{1}{2}$ if $N S$ is a half-integer.

For the proof, see Appendix 3.7 
The range of $\gamma$ that includes the point $\gamma=0$ and in which (51) does not vanish is maximal if we set $\alpha=\alpha_{0} \equiv \frac{\gamma}{2}\left(N S(2 S+1-N S)-s_{\mathcal{o}}\left(s_{\mathcal{o}}+1\right)\right)$. Then $\operatorname{det}\left(\eta_{N}^{S}\left(\alpha_{0}\right)\right)>0$ for $|\gamma|<\hat{\gamma}_{S}$, where

$$
\widehat{\gamma}_{S}=\frac{\pi}{\left(N S-s_{\mathcal{o}}\right)\left(N S+s_{\mathcal{o}}+1\right)} .
$$

Since $\frac{1}{2} \eta_{N}^{S}(0)=1$ for $\gamma=0$, we conclude that $\eta_{N}^{S}\left(\alpha_{0}\right)$ is positive definite for $|\gamma|<\hat{\gamma}_{S}$. Thus, we have established the following statement.

Proposition 3. The Hamiltonian $\mathrm{H}_{N}^{S}$ given by (41) is quasi-Hermitian for any choice of the coupling constants $b_{n, s}$ provided that $|\gamma|<\hat{\gamma}_{S}$, where $\hat{\gamma}_{S}$ is given by (52).

\section{CONCLUSion}

It is well known that for a given quasi-Hermitian operator $\mathrm{H}$ there are many metric operators $[\mathrm{SGH}, \mathrm{Be}, \mathrm{M} 2$. In the physical literature on non-Hermitian Hamiltonians, the one most frequently discussed is the operator $\eta_{0}$ considered in Remark 1 For the case of $\mathrm{H}$ having a simple spectrum, a generalization of $\eta_{0}$ to an operator of type (9) was given in $[\mathrm{ZG}$. In the present paper, we gave the most general form of a metric operator for a finite-dimensional quasi-Hermitian operator $\mathrm{H}$, not assuming its spectrum to be simple.

As an example of a compound operator (2) given by the sum of quasi-Hermitian operators, we studied the Hamiltonians (22) and (41) of an open $U_{q}\left(s l_{2}\right)$-invariant spin chain of spin $S$ and length $N$. For these Hamiltonians, we constructed two symmetrizing operators $\eta_{N}^{ \pm}$in terms of products of local R-matrices (it should be noted that similar products appeared in a different context in [TV]). From the operators $\eta_{N}^{ \pm}$we built a multi-parameter family of metric operators. These metric operators are universal, i.e., independent of the coupling constants, and thus nondynamical; i.e., their construction does not require the knowledge of the eigenvectors of a Hamiltonian.

By optimizing the value of the free parameter in a one-parameter subfamily of universal metric operators, we obtained an estimate (52) on the range of the deformation parameter $\gamma$ in which the Hamiltonians under consideration are quasi-Hermitian. Note that this range is in general narrower than the ranges of $\gamma$ for which the short chains considered in Subsections 2.2 and 2.3 have real spectra. We expect that better estimates of the quasi-Hermiticity range can be obtained by using the multi-parameter family (49).

It is worthy of mention that the most general family (41) of Hamiltonians includes, in particular, all known (see, e.g., B2]) integrable $U_{q}\left(s l_{2}\right)$-invariant spin chains with nearest-neighbor interaction: the XXZ model of spin $S$, the Temperley-Lieb spin chain of spin $S$, and, for spin 1, the spin chain generated by the Izergin-Korepin R-matrix. So our construction of the metric operators also applies to these cases.

We conclude with several remarks on the "experimental" data obtained in Subsections 2.2 and 2.3 for the ranges of $\gamma$ in which the Hamiltonian (22) has a real spectrum. First, it is very interesting to note that the value of $\widetilde{\gamma}_{\frac{1}{2}, 0}$ in (40) for an alternating XXZ chain of spin $\frac{1}{2}$ is exactly the same as the boundary of the quasi-Hermiticity range for a homogeneous XXZ chain of spin $\frac{1}{2}$ found in $\mathrm{KW}$ by means of the path basis technique. Actually, the results for short chains seem to indicate that, for given $S$ and $N$, the alternating chain $\left(a_{1}=-a_{2}=a_{3}=-a_{4}=\cdots\right)$ is the most non-Hermitian one, at least in the subclass of chains with a two-periodic coupling $\left(a_{2 n+1}=a_{1}, a_{2 n}=a_{2}\right)$. Thus, we have a reason to expect that Conjecture 2 may be true not only for alternating but also for two-periodic chains and, possibly, even for arbitrary ones.

Finally, we remind the reader that in the general $N=3$ case and in the two-periodic $N=4$ case, the spectra are always real if all coupling constants are positive. This observation is supported by numerical checks in a number of other cases. It is thus tempting to suggest the following. 
Conjecture 3. For $|\gamma|<\frac{\pi}{2 S}$, the Hamiltonian (22) of a spin chain with inhomogeneous coupling has a real spectrum if all $a_{n}$ are strictly positive.

\section{§3. Appendix}

3.1. Proof of Proposition 1, The spectral resolutions of a quasi-Hermitian operator $\mathrm{H}$ and its adjoint are $\mathbf{H}=\sum_{j=1}^{d^{\prime}} \lambda_{j} \mathfrak{P}_{j}, \mathbf{H}^{*}=\sum_{j=1}^{d^{\prime}} \lambda_{j} \mathfrak{P}_{j}^{*}$, where the $\mathfrak{P}_{j}=\sum_{k=1}^{\mu_{j}} \mathrm{P}_{j, k}$ are the projectors onto the subspaces $\mathfrak{H}_{j}$. Hence,

$$
\mathfrak{P}_{j}=\prod_{n \neq j}^{d^{\prime}} \frac{\mathrm{H}-\lambda_{n} 1}{\lambda_{j}-\lambda_{n}}, \quad \mathfrak{P}_{j}^{*}=\prod_{n \neq j}^{d^{\prime}} \frac{\mathrm{H}^{*}-\lambda_{n} 1}{\lambda_{j}-\lambda_{n}} .
$$

Relation (11) implies that $\eta \mathrm{H}^{n}=\left(\mathrm{H}^{*}\right)^{n} \eta$ for all $n \in \mathbb{N}$. Therefore, $\eta f(\mathrm{H})=(f(\mathrm{H}))^{*} \eta$, where $f(t)$ is an arbitrary polynomial with real coefficients. Along with (53), this shows that a positive definite operator $\eta$ is a metric operator for $\mathrm{H}$ if and only if

$$
\eta \mathfrak{P}_{j}=\mathfrak{P}_{j}^{*} \eta, \quad j=1, \ldots, d^{\prime} .
$$

As the basis of $\mathfrak{H}$, we take a naturally ordered set $\left\{\omega_{1,1}, \ldots, \omega_{1, \mu_{1}}, \omega_{2,1}, \ldots, \omega_{d^{\prime}, \mu_{d^{\prime}}}\right\}$. Then, by (8), we have $\widetilde{O}\left(\mathfrak{P}_{j}\right)=G E_{j}$ and $\widetilde{O}\left(\mathfrak{P}_{j}^{*}\right)=E_{j} G$, where $E_{j}$ is a diagonal matrix with $\mu_{j}$ consecutive entries equal to 1 and the others equal to 0 ; the identity matrix has the resolution $E=\sum_{j=1}^{d^{\prime}} E_{j}$. Using (6), we find that $\widetilde{O}\left(\eta \mathfrak{P}_{j}\right)=\widetilde{O}(\eta) E_{j}$ and $\widetilde{O}\left(\mathfrak{P}_{j}^{*} \eta\right)=$ $E_{j} \widetilde{O}(\eta)$. Therefore, (54) is true if and only if $\widetilde{O}(\eta)$ commutes with $E_{j}$ for all $j$, that is, if and only if $\widetilde{O}(\eta)$ is a block diagonal matrix. The second relation in (7) implies that $O\left(\eta^{-1}\right)$ is inverse to $\widetilde{O}(\eta)$ and so it is also a block diagonal matrix, whence equations (9) follow. The Hermitian nature of $\eta$ is equivalent to $(\widetilde{O}(\eta))^{*}=(\widetilde{O}(\eta))$, which implies that the blocks $\Phi_{j}$ in (9) must be Hermitian. Since $\eta$ is invertible, it is positive definite whenever $\eta^{-1}$ is so. The latter condition requires, in particular, that $\left\langle x_{j}, \eta^{-1} x_{j}\right\rangle>0$ for any nonzero vector $x_{j} \in \mathfrak{H}_{j}$. This is equivalent to $\sum_{k, n=1}^{\mu_{j}}\left(\Phi_{j}^{-1}\right)_{k n} \overline{\beta_{k}} \beta_{n}>0$, where the $\beta_{k} \equiv\left\langle\omega_{j, k}, x_{j}\right\rangle$ can be arbitrary (but not all zero). Thus, $\Phi_{j}^{-1}$ must be positive definite, and hence so must be $\Phi_{j}$.

To prove part b), we fix some bases $\left\{\omega_{j, k}^{\circ}\right\}$ in the subspaces $\mathfrak{H}_{j}$. Consider $\eta$ and $\eta^{-1}$ given by (9) with some matrices $\Phi_{j}^{\circ}$. Let $U_{j}$ be unitary matrices such that $\Phi_{j}=U_{j} \Phi_{j}^{\circ} U_{j}^{-1}$ are diagonal. Then, introducing new basis vectors $\omega_{j, k}=\sum_{n}\left(U_{j}^{-1}\right)_{k n} \omega_{j, n}^{\mathrm{o}}$, we see that, in the new basis, the symbol $O\left(\eta^{-1}\right)$ becomes a diagonal matrix. The second relation in (17) implies that $\widetilde{O}(\eta)$ also becomes a diagonal matrix. It remains to use formulas (8) to obtain equations (10).

3.2. Projectors $\mathrm{P}^{S, s}$. Let $q=e^{i \gamma}$. The algebra (16) has the following Casimir element:

$$
C=\frac{1}{2}(E F+F E)-\frac{\cos \gamma}{4 \sin ^{2} \gamma}\left(K-K^{-1}\right)^{2} .
$$

Its value in an irreducible representation $V^{S}$ is $\pi_{S}(C)=[S][S+1]$, where the $q$-numbers are defined as $[t] \equiv \frac{\sin \gamma t}{\sin \gamma}$. The tensor Casimir element is an operator in $V^{S} \otimes V^{S}$ given by

$$
\begin{aligned}
C^{S, S} & =\left(\pi_{S} \otimes \pi_{S}\right) \Delta(C)=\left(\pi_{S} \otimes \pi_{S}\right)\left((K E) \otimes\left(F K^{-1}\right)+\left(F K^{-1}\right) \otimes(K E)\right. \\
& \left.+\frac{1}{2 \sin ^{2} \gamma}\left(\left(1 \otimes 1+K^{2} \otimes K^{-2}\right) \cos \gamma-\left(1 \otimes K^{-2}+K^{2} \otimes 1\right) \cos (\gamma(2 S+1))\right)\right) .
\end{aligned}
$$

Obviously, we have $\left[C^{S, S},\left(\pi_{S} \otimes \pi_{S}\right)(\Delta(X))\right]=0$ for any $X \in U_{q}\left(s l_{2}\right)$. Furthermore, we have

$$
\left[C_{n, n+1}^{S, S}, \pi_{S}^{\otimes N}\left(\Delta^{(N-1)}(X)\right)\right]=0
$$


for any $X$ and $n=1, \ldots, N-1$. This can be verified by evaluating $\pi_{S}^{\otimes N}\left(\Delta_{N-1, n}(Y)\right)$, where $Y=\left[C_{n},\left(\Delta^{(N-2)}(X)\right)\right]=0$.

With respect to the involution (19), the tensor Casimir element is not Hermitian but is a symmetrizable operator,

$$
\left(\mathrm{C}^{S, S}\right)^{*}=\mathrm{C}_{q^{-1}}^{S, S}=\mathbb{P} C^{S, S} \mathbb{P} .
$$

Here $\mathrm{C}_{q^{-1}}^{S, S}$ is the tensor Casimir element of the algebra $U_{q^{-1}}\left(s l_{2}\right)$ (which is obtained by the mapping $\left.E \rightarrow E, F \rightarrow F, K \rightarrow K^{-1}, q \rightarrow q^{-1}\right)$.

The projectors $\mathrm{P}^{S, s}$ can be constructed as follows (see, e.g., [B1]):

$$
\mathrm{P}^{S, s}=\prod_{\substack{l=0 \\ l \neq s}}^{2 S} \frac{\mathrm{C}^{S, S}-[l][l+1]}{[s-l][s+l+1]}
$$

In particular, for $S=\frac{1}{2}$ we have

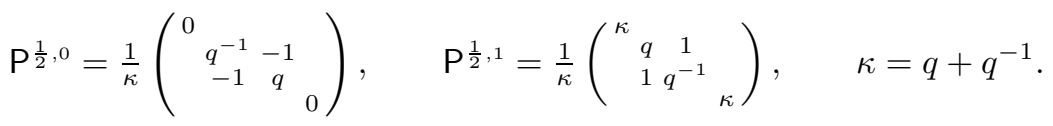

Note that the matrix entries of $\mathrm{P}^{S, s}$ can have singularities at some values of $\gamma$. This means that at these points the Gram matrix of the basis of $V^{S} \otimes V^{S}$ is not invertible (cf. equation (8)) and some basis vectors become linearly dependent. We shall exclude such values of $\gamma$ from consideration.

Since $\mathrm{P}^{S, s}$ are polynomials (with real coefficients) in $\mathrm{C}^{S, S}$, they satisfy the same relations (57) and (58), i.e.,

$$
\left[\mathrm{P}_{n, n+1}^{S, s}, \pi_{S}^{\otimes N}\left(\Delta^{(N-1)}(X)\right)\right]=0, \quad\left(\mathrm{P}^{S, s}\right)^{*}=\mathrm{P}_{q^{-1}}^{S, s}=\mathbb{P} \mathrm{P}^{S, s} \mathbb{P} .
$$

The first identity in the second relation implies, in particular, that $\left.\widetilde{\omega}_{s, k} \simeq \omega_{s, k}\right|_{q \rightarrow \bar{q}}=\bar{\omega}_{s, k}$, where $\simeq$ means equality up to a normalization (recall that in general the $\widetilde{\omega}$ are not normalized; cf. Remark 3). Using this relation and formulas (8), we can write out a more explicit expression for $\mathrm{P}^{S, s}$ :

$$
\mathrm{P}^{S, s}=\sum_{k=-s}^{s} \mathrm{P}_{s, k}=\sum_{k=-s}^{s} \frac{1}{\kappa_{s, k}} \omega_{s, k} \bar{\omega}_{s, k}^{\dagger},
$$

where $\kappa_{s, k}=\left\langle\bar{\omega}_{s, k}, \omega_{s, k}\right\rangle=\left\|\omega_{s, k}\right\|_{q \in \mathbb{R}}^{2}$, which is square of the norm of $\omega_{s, k}$ for $q \in \mathbb{R}$. Consider, for instance, the case of $s=0$. The corresponding submodule $V^{0}$ is one-dimensional and it is easy to find its basis vector $\omega_{0,0}$ (which is annihilated by both $\left(\pi_{S} \otimes \pi_{S}\right) \Delta(E)$ and $\left.\left(\pi_{S} \otimes \pi_{S}\right) \Delta(F)\right)$,

$$
\omega_{0,0}=\sum_{k=-S}^{S} \frac{(-1)^{S-k} q^{-k}}{\sqrt{2 S+1}} \omega_{k} \otimes \omega_{-k},
$$

so that $\kappa_{0,0}=\frac{[2 S+1]}{2 S+1}$. Substituting $\omega_{0,0}$ in (61) and identifying $\omega_{k} \simeq e_{S+1-k}$, where $e_{k}$ is a vector in $\mathbb{C}^{2 S+1}$ such that $\left(e_{k}\right)_{r}=\delta_{k r}$, we obtain the following matrix form of $\mathrm{P}^{S, 0}$ :

$$
\mathrm{P}^{S, 0}=\sum_{m, n=1}^{2 S+1} \frac{(-1)^{m+n} q^{m+n-2 S-2}}{[2 S+1]} E_{m, n} \otimes E_{2 S+2-m, 2 S+2-n},
$$

where the $E_{m, n}$ are matrices of size $2 S+1$ such that $\left(E_{m, n}\right)_{k l}=\delta_{m k} \delta_{n l}$. 
3.3. Minimal polynomial $\mathcal{P}_{a_{1}, a_{2}}^{S, 0}$. For $\mathrm{H}=a_{1} \mathrm{P}_{12}^{S, 0}+a_{2} \mathrm{P}_{23}^{S, 0}$, we have

$$
\mathrm{H}^{2}=a_{1}^{2} \mathrm{P}_{12}^{S, 0}+a_{2}^{2} \mathrm{P}_{23}^{S, 0}+a_{1} a_{2}\left(\mathrm{P}_{12}^{S, 0} \mathrm{P}_{23}^{S, 0}+\mathrm{P}_{23}^{S, 0} \mathrm{P}_{12}^{S, 0}\right) \text {. }
$$

Multiplying this expression by $\mathrm{H}$ and using (25), we find

$$
\mathrm{H}^{3}=a_{1}^{3} \mathrm{P}_{12}^{S, 0}+a_{2}^{3} \mathrm{P}_{23}^{S, 0}+a_{1} a_{2}\left(a_{1}+a_{2}\right)\left(\mathrm{P}_{12}^{S, 0} \mathrm{P}_{23}^{S, 0}+\mathrm{P}_{23}^{S, 0} \mathrm{P}_{12}^{S, 0}\right)+\mu_{S} a_{1} a_{2} \mathrm{H},
$$

whence $\mathrm{H}^{3}-\left(a_{1}+a_{2}\right) \mathrm{H}^{2}=\left(\mu_{S}-1\right) a_{1} a_{2} \mathrm{H}$. Thus, the minimal polynomial for $\mathbf{H}$ is (26)).

3.4. Coefficients $d_{k}^{S, s}$ for the minimal polynomials $\mathcal{P}_{a_{1}, a_{2}}^{S, s}$. We denote $[t] \equiv \frac{\sin \gamma t}{\sin \gamma}$ and $\{t\} \equiv 2 \cos \gamma t$. The coefficients $d_{k}^{S, s}$ in (29) are given by

$$
\begin{aligned}
& S=1, s=1: d_{1}^{1,1}=\frac{1}{\{2\}^{2}}, \quad d_{2}^{1,1}=\left(\frac{\{3\}}{\{1\}\{2\}}\right)^{2} ; \\
& S=1, s=2: d_{1}^{1,2}=\frac{1}{\{2\}^{2}}, \quad d_{2}^{1,2}=\left(\frac{1}{\{2\}[3]}\right)^{2}, \quad d_{3}^{1,2}=1 \\
& S=\frac{3}{2}, s=1: d_{1}^{\frac{3}{2}, 1}=\left(\frac{[3]}{\{2\}[5]}\right)^{2}, \quad d_{2}^{\frac{3}{2}, 1}=\frac{1}{\{2\}^{2}}, \quad d_{3}^{\frac{3}{2}, 1}=\left(\frac{[2][6]-1}{[4][5]}\right)^{2} ; \\
& S=\frac{3}{2}, s=2: d_{1}^{\frac{3}{2}, 2}=\frac{1}{\{3\}^{2}}, \quad d_{2}^{\frac{3}{2}, 2}=\frac{1}{\{2\}^{2}}, \quad d_{3}^{\frac{3}{2}, 2}=\left(\frac{\{5\}}{\{2\}\{3\}}\right)^{2}, \quad d_{4}^{\frac{3}{2}, 2}=\left(\frac{[5]-2}{\{2\}\{3\}}\right)^{2} ; \\
& S=\frac{3}{2}, s=3: d_{1}^{\frac{3}{2}, 3}=\frac{1}{\{3\}^{2}}, \quad d_{2}^{\frac{3}{2}, 3}=\left(\frac{\{1\}}{\{3\}[5]}\right)^{2}, \quad d_{3}^{\frac{3}{2}, 3}=\left(\frac{1}{\{2\}\{3\}[5]}\right)^{2}, \quad d_{4}^{\frac{3}{2}, 3}=1 .
\end{aligned}
$$

The minimal positive solutions $\gamma_{S, s}$ of the equation $d_{1}^{S, s}=1$ are the following:

$$
\gamma_{1,1}=\gamma_{1,2}=\frac{\pi}{6}, \quad \gamma_{\frac{3}{2}, 1}=\frac{\pi}{7}, \quad \gamma_{\frac{3}{2}, 2}=\gamma_{\frac{3}{2}, 3}=\frac{\pi}{9} .
$$

Let us mention in passing an interesting pattern in the minimal positive solutions of the equation $d_{k}^{S, s}=1$ for $s=2 S$ : we have $\gamma_{1,2}^{\{1\}}=\frac{\pi}{6}, \gamma_{1,2}^{\{2\}}=\frac{\pi}{5}$, and $\gamma_{\frac{3}{2}, 3}^{\{1\}}=\frac{\pi}{9}, \gamma_{\frac{3}{2}, 3}^{\{2\}}=\frac{\pi}{8}$, $\gamma_{\frac{3}{2}, 3}^{\{3\}}=\frac{\pi}{7}$.

3.5. Universal R-matrix. In [D1, Drinfeld showed that relations (43) and (44) are satisfied for $R^{+}$and $R^{-} \equiv \mathbb{P}\left(R^{+}\right)^{-1} \mathbb{P}$, where $R^{+}$is given by

$$
R^{+}=q^{H \otimes H} \sum_{n=0}^{\infty} \frac{q^{\frac{1}{2}\left(n^{2}-n\right)}}{\prod_{k=1}^{n}[k]_{q}}\left(\left(q-q^{-1}\right) F \otimes E\right)^{n} q^{H \otimes H} .
$$

Here $H$ is related to $K$ via $K=q^{H}$. Relations (43)-(44) imply the Yang-Baxter equation

$$
R_{12}^{ \pm} R_{13}^{ \pm} R_{23}^{ \pm}=R_{23}^{ \pm} R_{13}^{ \pm} R_{12}^{ \pm}
$$

Note that $\left.R^{+}\right|_{q \rightarrow q^{-1}}=\left(R^{+}\right)^{-1}$. Therefore, for $|q|=1$ we have

$$
\left(R^{+}\right)^{*}=R^{-} .
$$

3.6. Proof of Proposition 2, We introduce an operation $\Delta^{ \pm} \equiv R^{ \pm} \Delta$ and define its action on $X \in U_{q}\left(s l_{2}\right)^{\otimes N}$ by the following formula: $\Delta_{N, n}^{ \pm}(X) \equiv R_{n, n+1}^{ \pm} \Delta_{N, n}(X)$ (recall that $\Delta_{N, n}$ was defined after equation (23)).

Lemma 2. a) $\Delta^{ \pm}$is coassociative, i.e.,

$$
\Delta_{2,1}^{ \pm} \circ \Delta^{ \pm}=\Delta_{2,2}^{ \pm} \circ \Delta^{ \pm}
$$

Therefore, a positive integral power of $\Delta^{ \pm}$can be defined in the same way as was done for $\Delta$, i.e.,

$$
\left(\Delta^{ \pm}\right)^{(N)}=\Delta_{N, n}^{ \pm} \circ\left(\Delta^{ \pm}\right)^{(N-1)} .
$$


The operations $\Delta^{+}$and $\Delta^{-}$are conjugate to each other in the following sense:

$$
\left(\Delta^{+}(X)\right)^{*}=\Delta^{-}\left(X^{*}\right)
$$

for any $X \in U_{q}\left(s l_{2}\right)$.

b) Equivalently, the symmetrizing operators (46) can be represented as follows:

$$
\eta_{N+1}^{ \pm}=\pi_{S}^{\otimes N+1}\left(\Delta_{N, n}^{ \pm}\left(\widetilde{\eta}_{N}^{ \pm}\right)\right)=\pi_{S}^{\otimes(N+1)}\left(\left(\Delta^{ \pm}\right)^{(N)}(1)\right),
$$

where the $\widetilde{\eta}_{N}^{ \pm}$are given by (46) with $R_{n m}^{ \pm}$instead of $\mathrm{R}_{n m}^{ \pm}$, and $\widetilde{\eta}_{1}^{ \pm} \equiv 1$.

In (69) and (71), $n$ can take any value from 1 to $N$.

Proof a). The coassociativity of $\Delta^{ \pm}$follows from the coassociativity of $\Delta$ along with the Yang-Baxter equation:

$$
\begin{aligned}
\Delta_{2,1}^{ \pm} \circ \Delta^{ \pm}(X) & =\Delta_{2,1}^{ \pm}\left(R^{ \pm} \Delta(X)\right) \stackrel{\left(\frac{44}{=}\right.}{=} R_{12}^{ \pm} R_{13}^{ \pm} R_{23}^{ \pm} \Delta_{2,1}(X) \\
& \stackrel{(66)}{=} R_{23}^{ \pm} R_{13}^{ \pm} R_{12}^{ \pm} \Delta_{2,2}(X) \stackrel{(43)}{=} \Delta_{2,2}^{ \pm}\left(R^{ \pm} \Delta(X)\right)=\Delta_{2,2}^{ \pm} \circ \Delta^{ \pm}(X) .
\end{aligned}
$$

Property (70) is checked easily:

$$
\left(\Delta^{+}(X)\right)^{*}=\left(R^{+} \Delta(X)\right)^{*} \stackrel{\sqrt[667]{=}}{=} \Delta^{\prime}\left(X^{*}\right) R^{-\stackrel{443]}{=}} R^{-} \Delta\left(X^{*}\right)=\Delta^{-}\left(X^{*}\right) .
$$

b) First, we shall prove the first identity in (71) by induction in the case of $n=N-1$. The base of induction, for $N=2$, holds by the definition of $\Delta^{ \pm}$and the relation $\Delta(1)=$ $1 \otimes 1$. The inductive step (which can be regarded as an extension of the lattice by an additional node) is checked as follows:

$$
\begin{aligned}
\eta_{N+1}^{ \pm} & \stackrel{(46)}{=} \stackrel{\mathrm{R}}{N+1}_{N}^{ \pm} \stackrel{\leftarrow}{\mathrm{R}}_{N}^{ \pm} \eta_{N-1}^{ \pm}=\mathrm{R}_{N, N+1}^{ \pm} \mathrm{R}_{N-1, N+1}^{ \pm} \ldots \mathrm{R}_{1, N+1}^{ \pm} \mathrm{R}_{N-1, N}^{ \pm} \ldots \mathrm{R}_{1 N}^{ \pm} \eta_{N-1}^{ \pm} \\
& =\mathrm{R}_{N, N+1}^{ \pm}\left(\mathrm{R}_{N-1, N+1}^{ \pm} \mathrm{R}_{N-1, N}^{ \pm} \ldots \mathrm{R}_{n, N+1}^{ \pm} \mathrm{R}_{n, N}^{ \pm} \ldots \mathrm{R}_{1, N+1}^{ \pm} \mathrm{R}_{1, N}^{ \pm}\right) \eta_{N-1}^{ \pm} \\
& \stackrel{(44)}{=} \pi_{S}^{\otimes(N+1)}\left(R_{N, N+1}^{ \pm} \Delta_{N, N}\left(R_{N-1, N}^{ \pm} \ldots R_{1 N}^{ \pm}\right) \tilde{\eta}_{N-1}^{ \pm}\right) \\
& =\pi_{S}^{\otimes(N+1)}\left(R_{N, N+1}^{ \pm} \Delta_{N, N}\left(\stackrel{\overleftarrow{R}}{R_{N}} \tilde{\eta}_{N-1}^{ \pm}\right)\right) \stackrel{(46)}{=} \pi_{S}^{\otimes(N+1)}\left(\Delta_{N, N}^{ \pm}\left(\widetilde{\eta}_{N}^{ \pm}\right)\right),
\end{aligned}
$$

whence $\eta_{N+1}^{ \pm}=\pi_{S}^{\otimes(N+1)}\left(\Delta_{N, N}^{ \pm} \circ \Delta_{N-1, N-1}^{ \pm} \circ \cdots \circ \Delta_{1,1}^{ \pm}\left(\tilde{\eta}_{1}^{ \pm}\right)\right) \stackrel{(696)}{=} \pi_{S}^{\otimes(N+1)}\left(\left(\Delta^{ \pm}\right)^{(N)}(1)\right)$. That is, we have proved that $\eta_{N+1}^{ \pm}$is equal to the last expression in (71). In its turn, the latter is equal to the middle expression in (71), because $n$ in the definition (69) can be arbitrary from 1 to $N$. This completes the proof of Lemma 1 .

Proof of Proposition 2. We commence by proving part b). Choosing $n=1$ in (71), we can write $\eta_{N+1}^{ \pm}$as follows: $\eta_{N+1}^{ \pm}=\pi_{S}^{\otimes N+1}\left(\left(\Delta^{ \pm}\right)^{(N)}(1)\right)=\pi_{S}^{\otimes N+1}\left(\Delta_{N, 1}^{ \pm} \circ \Delta_{N-1,1}^{ \pm} \circ \cdots \circ\right.$ $\left.\Delta_{1,1}^{ \pm}(1)\right)$. Then expressions (47) can be obtained by an induction similar to that in the proof of Lemma 1, but this time one should use the first relation in (44).

Relation (48) in part c) of Proposition 2 is an immediate consequence of applying relation (70) to formula (71).

To prove part a) of Proposition 2, first we show that the $\eta_{N}^{ \pm}$are symmetrizing operators for the tensor Casimir element:

$$
\begin{aligned}
\eta_{N}^{ \pm} \mathrm{C}_{n, n+1} & \stackrel{\stackrel{(71)}{=} \pi_{S}^{\otimes N}}{\otimes_{n}}\left(R_{n, n+1}^{ \pm} \Delta_{N-1, n}\left(\widetilde{\eta}_{N-1}^{ \pm} C_{n}\right)\right)=\pi_{S}^{\otimes N}\left(R_{n, n+1}^{ \pm} \Delta_{N-1, n}\left(C_{n} \widetilde{\eta}_{N-1}^{ \pm}\right)\right) \\
& \stackrel{(71]}{=} \mathrm{R}_{n, n+1}^{ \pm} \mathrm{C}_{n, n+1}\left(\mathrm{R}_{n, n+1}^{ \pm}\right)^{-1} \eta_{N}^{ \pm} \stackrel{(43)}{=} \mathrm{C}_{n+1, n} \eta_{N}^{ \pm} .
\end{aligned}
$$

Therefore, the $\eta_{N}^{ \pm}$are symmetrizing operators also for an arbitrary polynomial in $\mathrm{C}_{n, n+1}$ with real coefficients. Consequently, taking formula (59) into account, we conclude that relation (42) is true. Thus, Proposition 2 is proved. 
3.7. Proof of Lemma 1. The bialgebra defined by relations (16)-(17) turns into a Hopf algebra if the antipode (an antihomomorphism) is defined as follows: $\mathcal{S}(E)=-q^{-1} E$, $\mathcal{S}(F)=-q F, \mathcal{S}(K)=K^{-1}$.

The R-matrix (65) has the following form: $R^{+}=\sum_{a} r_{a}^{(1)} \otimes r_{a}^{(2)}$. Consider the element $\chi=K^{2}\left(\sum_{a} \mathcal{S}\left(r_{a}^{(1)}\right) r_{a}^{(2)}\right)$. From the results of [D2], it follows that $\chi$ is a central element, which acquires the value $q^{-2 S(S+1)}$ on an irreducible module $V^{S}$, and that $\chi$ satisfies the relation:

$$
\chi_{1} \chi_{2} \Delta\left(\chi^{-1}\right)=\left(R^{-}\right)^{-1} R^{+} .
$$

We prove that

$$
\chi_{1} \ldots \chi_{N} \Delta^{(N-1)}\left(\chi^{-1}\right)=\left(\widetilde{\eta}_{N}^{-}\right)^{-1} \tilde{\eta}_{N}^{+} .
$$

For $N=2$, this relation coincides with (72). For $N \geq 3$, it is verified by induction:

$$
\begin{aligned}
& \chi_{1} \ldots \chi_{N+1} \Delta^{(N)}\left(\chi^{-1}\right) \stackrel{[727}{=}\left(R_{12}^{-}\right)^{-1} R_{12}^{+} \Delta_{N, 1}\left(\chi_{1} \ldots \chi_{N} \Delta^{(N-1)}\left(\chi^{-1}\right)\right) \\
& \stackrel{[73}{=}\left(R_{12}^{-}\right)^{-1} R_{12}^{+} \Delta_{N, 1}\left(\left(\widetilde{\eta}_{N}^{-}\right)^{-1}\right) \Delta_{N, 1}\left(\widetilde{\eta}_{N}^{+}\right)=\left(\Delta_{N, 1}^{-}\left(\widetilde{\eta}_{N}^{-}\right)\right)^{-1} \Delta_{N, 1}^{+}\left(\widetilde{\eta}_{N}^{+}\right) \stackrel{(719}{=}\left(\widetilde{\eta}_{N+1}^{-}\right)^{-1} \widetilde{\eta}_{N+1}^{+} .
\end{aligned}
$$

If $q$ is not a root of unity, the center of the algebra $U_{q}\left(s l_{2}\right)$ is generated by the Casimir element (55). Therefore, there exists a function $\varphi_{q}$ such that $\chi=\varphi_{q}(C)$. Consequently, in each irreducible submodule $V^{s} \subset\left(V^{S}\right)^{\otimes N}$ the operator $\Delta^{(N-1)}(\chi)=\varphi\left(\Delta^{(N-1)}(C)\right)$ acts as multiplication by $q^{-2 s(s+1)}$. Along with formula (73), this implies that

$$
\left(\eta_{N}^{-}\right)^{-1} \eta_{N}^{+}=\sum_{s=s_{\mathcal{o}}}^{N S} q^{2 s(s+1)-2 N S(S+1)} \mathcal{P}_{s},
$$

where $\mathcal{P}_{s}$ denotes the projector of rank $\nu_{s}(2 s+1)$ onto the reducible invariant subspace $\oplus^{\nu_{s}} V^{s} \subset\left(V^{S}\right)^{\otimes N}$.

Using (74), we derive formula (51):

$$
\begin{aligned}
\operatorname{det}\left(e^{i \alpha} \eta_{N}^{+}+e^{-i \alpha} \eta_{N}^{-}\right) & =\operatorname{det}\left(\eta_{N}^{-}\right) \operatorname{det}\left(e^{i \alpha}\left(\eta_{N}^{-}\right)^{-1} \eta_{N}^{+}+e^{-i \alpha} 1\right) \\
& \stackrel{(74)}{=} \operatorname{det}\left(\sum_{s=s_{\mathcal{o}}}^{N S}\left(e^{i \alpha} q^{2 s(s+1)-2 N S(S+1)}+e^{-i \alpha}\right) \mathcal{P}_{s}\right) \\
& =\rho_{N, S} \prod_{s=s_{\mathcal{o}}}^{S N}\left(e^{i \alpha} q^{s(s+1)-N S(S+1)}+e^{-i \alpha} q^{N S(S+1)-s(s+1)}\right)^{\nu_{s}(2 s+1)},
\end{aligned}
$$

where $\rho_{N, S} \equiv \prod_{s=s_{0}}^{S N} q^{\nu_{s}(2 s+1)(s(s+1)-N S(S+1))}=1$, which follows from (74) and the relation $\operatorname{det} \eta_{N}^{ \pm}=1\left(\right.$ note that $\left.\operatorname{det} \mathrm{R}^{ \pm}=1\right)$.

\section{ACKNowledgments}

This work was started during the author's visit (supported by the Swiss National Science Foundation under grant 200020-121675) to the Mathematics Department, University of Geneva, and completed during a visit (supported by the Alexander von Humboldt Foundation) to DESY, Hamburg. The work was also supported in part by the Russian Foundation for Basic Research (grant nos. 07-02-92166, 08-01-00638, 09-01-12150, 09-01-93108).

The author thanks A. Alekseev, A. Fring, G. von Gehlen, P. Kulish, and V. Tarasov for useful remarks. 


\section{REFERENCES}

[AF] P. E. G. Assis and A. Fring, Metrics and isospectral partners for the most generic cubic PTsymmetric non-Hermitian Hamiltonian, J. Phys. A 41 (2008), 244001, 18 pp. MR2455799 (2009g:81052)

[BB1] M. T. Batchelor and M. N. Barber, Spin-s quantum chains and Temperley-Lieb algebras, J. Phys. A 23 (1990), L15-L21. MR1034619 (91c:82026)

[BB2] C. M. Bender and S. Boettcher, Real spectra in non-Hermitian Hamiltonians having PT symmetry, Phys. Rev. Lett. 80 (1998), 5243-5246. MR1627442 (99b:81043)

[Be] C. M. Bender, Making sense of non-Hermitian Hamiltonians, Rep. Progr. Phys. 70 (2007), 9471018. MR 2331294(2008h:81045)

[BBJ] C. M. Bender, D. C. Brody, and H. F. Jones, Complex extension of quantum mechanics, Phys. Rev. Lett. 89 (2002), 270401, 4 pp. MR1950305 (2004e:81078)

[BZ] D. Bessis and J. Zinn-Justin, 1993 (unpublished).

[B1] A. G. Bytsko, On integrable Hamiltonians for higher spin XXZ chain, J. Math. Phys. 44 (2003), 3698-3717. MR2003927 (2004g:82026)

[B2] - On higher spin $U_{q}\left(s_{2}\right)$-invariant R-matrices, Algebra i Analiz 17 (2005), no. 3, 24-46; English transl., St. Petersburg Math. J. 17 (2006), no. 3, 393-408. MR2167842 (2006g:17019)

[CS] J. L. Cardy and R. L. Sugar, Reggeon field theory on a lattice. 1, Phys. Rev. D 12 (1975), $2514-2522$.

[CF] O. A. Castro-Alvaredo and A. Fring, A spin chain model with non-Hermitian interaction: The Ising quantum spin chain in an imaginary field, J. Phys. A 42 (2009), 465211, 29 pp. MR2552019 (2010m:82013)

[Di] J. Dieudonné, Quasi-Hermitian operators, Proc. Internat. Sympos. Linear Spaces (Jerusalem, 1960), Jerusalem Acad. Press, Jerusalem, 1961, pp. 115-122. MR0187086 (32:4540)

[D1] V. G. Drinfel'd, Quantum groups, Zap. Nauchn. Sem. Leningrad. Otdel. Mat. Inst. Steklov. (LOMI) 155 (1986), 18-49; English transl., J. Soviet Math. 41 (1988), no. 2, 898-915. MR:0869575 (88f:17017)

[D2] , On almost cocommutative Hopf algebras, Algebra i Analiz 1 (1989), no. 2, 30-46; English transl., Leningrad Math. J. 1 (1990), no. 2, 321-342. MR1025154(91b:16046)

[Ge] G. von Gehlen, Critical and off critical conformal analysis of the Ising quantum chain in an imaginary field, J. Phys. A 24 (1991), 5371-5400.

[He] H. Heuser, Über Eigenwerte und Eigenlösungen symmetrisierbarer finiter Operatoren, Arch. Math. 10 (1959), 12-20. MR0102020 (21:816)

[Ho] T. Hollowood, Solitons in affine Toda field theories, Nuclear Phys. B 384 (1992), 523-540. MR $1188363(94 \mathrm{e}: 81290)$

[IK] A. G. Izergin and V. E. Korepin, The inverse scattering method approach to the quantum Shabat-Mikhailov model, Comm. Math. Phys. 79 (1981), 303-316. MR0627054 (83c:81092)

$[\mathrm{KR}]$ A. N. Kirillov and N. Yu. Reshetikhin, Representations of the algebra $U_{q}($ sl(2)), q-orthogonal polynomials and invariants of links, Infinite-Dimensional Lie Algebras and Groups (LuminyMarseille, 1988), Adv. Ser. Math. Phys., vol. 7, World Sci. Publ., Teaneck, NJ, 1989, pp. 285-339. MR:1026957 (90m:17022)

[KW] C. Korff and R. A. Weston, PT symmetry on the lattice: The quantum group invariant XXZ spin chain, J. Phys. A 40 (2007), 8845-8872. MR2344527(2008i:82023)

$[\mathrm{Ku}]$ P. P. Kulish, On spin systems related to the Temperley-Lieb algebra, J. Phys. A 36 (2003), L489-L493. MR2006441 (2004h:82029)

[KS] P. P. Kulish and A. A. Stolin, Deformed Yangians and integrable models, Czechoslovak. J. Phys. 47 (1997), 1207-1212. MR 1608809 (99g:81092)

[M1] A. Mostafazadeh, Pseudo-Hermiticity versus PT symmetry. III. Equivalence of pseudo-Hermiticity and the presence of antilinear symmetries, J. Math. Phys. 43 (2002), 3944-3951. MR 1915636(2003i:81234)

[M2] , Pseudo-Hermitian quantum mechanics, arXiv:0810.5643 [hep-th].

[Re] W. T. Reid, Symmetrizable completely continuous linear transformations in Hilbert space, Duke Math. J. 18 (1951), 41-56. MR0045314(13:564b)

[SGH] F. G. Scholtz, H. B. Geyer, and F. J. W. Hahne, Quasi-Hermitian operators in quantum mechanics and the variational principle, Ann. Physics 213 (1992), 74-101. MR.1144600 (94a:81028)

[S1] J. P. O. Silberstein, Symmetrisable operators, J. Austral. Math. Soc. 2 (1962), 381-402. MR.0149299 (26:6789)

[S2] Symmetrisable operators. II. Operators in a Hilbert space H, J. Austral. Math. Soc. 4 (1964), 15-30. MR0162138 (28:5337) 
[TV] A. N. Varchenko and V. O. Tarasov, Jackson integral representations of solutions of the quantized Knizhnik-Zamolodchikov equation, Algebra i Analiz 6 (1994), no. 2, 90-137; English transl., St. Petersburg Math. J. 6 (1995), no. 2, 275-313. MR1290820(96f:81052a)

[Wi] E. P. Wigner, Normal form of antiunitary operators, J. Math. Phys. 1 (1960), 409-413. MR 0117557 (22:8334)

[Za] A. C. Zaanen, Uber vollstetige symmetrische und symmetrisierbare Operatoren, Nieuw Arch. Wiskunde (2) 22 (1943), 57-80. MR0015661 (7:453g)

[ZG] M. Znojil and H. B. Geyer, Construction of a unique metric in quasi-Hermitian quantum mechanics: nonexistence of the charge operator in a $2 \times 2$ matrix model, Phys. Lett. B 640 (2006), 52-56. MR2245629 (2007b:81079)

St. Petersburg Branch, Steklov Mathematical Institute, Russian Academy of Sciences, 27 Fontanka, St. Petersburg 191023, Russia

E-mail address: bytsko@pdmi.ras.ru

Received 18/DEC/2009

Translated by THE AUTHOR 